\title{
Analisis of leptospirosis lethal cases in Lviv region
}

\author{
Olena Zubach* and Alexandr Zinchuk \\ Department of infectious diseases, Danylo Halytsky Lviv National Medical University, Lviv, Ukraine
}

\section{Objective}

Study of the structure of lethal cases in patients diagnosed with leptospirosis in the Lviv Region.

\section{Introduction}

Mortality rate of leptospirosis in Ukraine remains high year after year. The study of the peculiarities of lethal cases over a long period enables researchers to specify possible mechanisms of infection which cause the development of the severest cases of leptospirosis and to prevent disease emergence by applying adequate preventive measures.

\section{Methods}

We have analyzed case reports of patients diagnosed with leptospirosis, who were treated and died in Lviv Regional Clinical Hospital of Infectious Diseases from 1987-2016.

\section{Results}

Over the last 30 years, 942 patients with leptospirosis were treated in Lviv Regional Clinical Hospital of Infectious Diseases, and 125 of them died. The mortality rate was $13.27 \%$. Men died twice as often as women $-83(66.4 \%)$ and $42(33.6 \%)$, respectively, $p<0.001$. The average age at death was $56.5 \pm 11.98$ years old. Women died at an older age $(59.62 \pm 9.6)$ as compared to men $(54.93 \pm 12.78), \mathrm{p}<0.05$. Patients who died from leptospirosis were admitted into the hospital on the $5.81 \pm 2.31$ day, which is considered to be a late admission. The average length of stay in the inpatient department was $6.21 \pm 6.54$ days. Rural residents died much more often, 78 cases $(62.4 \%)$ compared with city residents, 47 cases $(37.6 \%), \mathrm{p}<0.01$. The mortality from leptospirosis was lowest during the summer months $(6.79 \%)$ compared to winter $(19.85 \%, \mathrm{p}<0.001)$, spring $(23.81 \%, \mathrm{p}<0.001)$ and autumn $(13.78 \%, \mathrm{p}<0.01)$ months. It should be noted that the mortality during the autumn-summer period was significantly lower $(10 \%)$ than during the spring-winter period $(21.76 \%, p<0.001)$. The causative agent was verified by microscopic agglutination test and lysis in $66(52.8 \%)$ of the deceased patients, and in 59 individuals $(47.2 \%)$ the agent could not be identified due to early period of serological investigation, when no anti-leptospirosis antibodies had been produced so far. In these cases the diagnosis of leptospirosis was based on typical clinical signs and epidemiological anamnesis. The main serogroups of leptospirae, which caused lethal cases, were L. icterohaemorrhagiae - 51 (40.8\%), L. grippotyphosa - 5 (4\%), L. kabura - 3 (2.4\%), mixed L. Icterohaemorrhagiae plus $L$. Grippotyphosa-3 (2.4\%), L. Cynopteri and L. Hebdomadis - 2(1.6\%) patients each. Epidemiological anamnesis could be determined in 84 $(67.2 \%)$ patients. Most often, patients associated the disease with the following factors: $45(36 \%)$ - with the presence of rats or mice-like rodents in a house, yard or workplace, $26(20.8 \%)$ - with agricultural work, where contact with rodents' feces was also possible, $5(4 \%)$ - with professional activity, $4(3.2 \%)$ - with fishing, and $4(3.2 \%)$ with swimming in water pools. In $41(32.8 \%)$ patients, leptospirosis could not be associated with any factor.

\section{Conclusions}

L.icterohaemorrhagiae still remains the most common cause of mortality of leptospirosis $-51(40.8 \%)$. In $56.8 \%$ of the cases, the disease was caused by contact with rodents. Over the last 30 years, men died more often of leptospirosis in the Lviv region than women $(p<0.001)$, whereas, the deceased women were considerably older than men $(\mathrm{p}<0.05)$. Rural residents died much more often than city residents $(\mathrm{p}<0.01)$. The highest mortality rate was recorded in the spring $-23.81 \%$, the lowest was recorded in summer $-6.79 \%$ (p $<0.001$ ). Active deratization measures help to reduce morbidity and mortality of leptospirosis.

We believe that the question regarding the accuracy of the final diagnosis of "leptospirosis" in $59(47.2 \%)$ dead patients is still under discussion, as the diagnosis was established based on clinical symptoms only, while PMA result was negative. After all, the clinical picture of the severe forms of leptospirosis is similar to the typical symptoms of hemorrhagic fever with renal syndrome and Crimean hemorrhagic fever.

\section{Keywords}

leptospirosis; lethality; surveillance

\section{Acknowledgments}

We would like to express our gratitude to the archive of Lviv Regional Clinical Hospital of Infectious Diseases and Especially Dangerous Infections Laboratory of Lviv Oblast Laboratory Center of the Ministry of Health of Ukraine for assistance with research.

\section{References}

1. Tubiana S., Mikulski M., Becam J., Lacassin F., Lefèvre P., Gourinat A-S., Goarant C., D'Ortenzio E. Risk Factors and Predictors of Severe Leptospirosis in New Caledonia. PLoS Neglected Tropical Diseases.2013;7(1):e1991.

2. Taylor AJ., Paris DH., Newton PN. A Systematic Review of the Mortality from Untreated Leptospirosis. PLoS Neglected Tropical Diseases. 2015; 25;9(6):e0003866.

\section{*Olena Zubach}

E-mail: dr_zubach@i.ua 